\title{
Metallic glass-formers in 2D exhibit the same scaling as in 3D between vibrational dynamics and structural relaxation
}

\author{
C A Massa ${ }^{1}$, D Leporini ${ }^{1,2}$ and F Puosi ${ }^{2}$ \\ ${ }^{1}$ Istituto per i Processi Chimico-Fisici-Consiglio Nazionale delle Ricerche (IPCF-CNR), via \\ G. Moruzzi 1, 56124 Pisa, Italy \\ ${ }^{2}$ Dipartimento di Fisica "Enrico Fermi”, Università di Pisa, Largo B. Pontecorvo 3, 56127 \\ Pisa, Italy \\ E-mail: francesco.puosi@df.unipi.it
}

\begin{abstract}
.
Glass-forming systems approaching their glass transition exhibit universal correlations between picosecond vibrational dynamics and long-time structural relaxation, which can be described by the same master curve in the bulk or confined conditions. In this work, we study at a fundamental level the effects of the reduction of spatial dimensionality on this phenomenon. We perform Molecular Dynamics simulations of a metallic glass-formers in two dimensions (2D). We show that in the supercooled regime particle localization in the cage and structural relaxation are blurred by long-wavelength fluctuations specific to low-dimensional systems. Once these effects are properly removed, we demonstrate that the fast dynamics and slow relaxation comply, without any adjustment, with same scaling between the structural relaxation time and the Debye-Waller factor, originally observed in three-dimensions (3D).
\end{abstract}




\section{Introduction}

In glass-forming liquids approaching the glass transition (GT), the huge slowing-down of structural relaxation and the increase of viscosity is accompanied by the localization of their constituent atoms and molecules, which are increasingly trapped by their surroundings, a phenomenon usually indicated as cage effect. Localization is quantified by the DebyeWaller (DW) factor $\left\langle u^{2}\right\rangle$, i.e., the mean square amplitude of particle motion on a picosecond timescale. In the last decade numerous evidences have been reported supporting the existence of a link between the long-time structural relaxation time $\tau_{\alpha}$ and the fast dynamics as denoted by $\left\langle u^{2}\right\rangle$. Correlations have been found in simulations of model systems such as polymers $[1,2,3]$, binary mixture [4], water [5, 6] and metallic liquids [7, 8] and validated with experimental data on several glass-formers $[1,9,10,3]$. Further, it was also shown that the fast dynamic property $\left\langle u^{2}\right\rangle$ encodes some basic aspects of the dynamic slowing-down as approaching the GT like the onset of mechanical rigidity[11], the development of dynamical heterogeneities (DHs) and the break-down of the Stokes-Einstein relation [12, 8] .

Remarkably, the correlation between fast dynamics and relaxations is not limited to bulk materials, where it was originally evidenced, but extends to non-bulk conditions. In simulations of supported thin films, Douglas and coworkers showed that, by considering the average dynamics of the film, the bulk-like scaling law between $\left\langle u^{2}\right\rangle$ and $\tau_{\alpha}$ is recovered with a slightly different scaling adjustable parameter [13]. Taking into account the strong gradient of mobility in the film via a layer resolved analysis, Leporini and coworkers improved on previous results and showed that the $\left\langle u^{2}\right\rangle-\tau_{\alpha}$ correlation is exactly the same of bulk, i.e., it is described by the same master curve with no adjustable parameters [14]. These findings suggest that the dynamics in thin films or confined materials and in their bulk counterpart are controlled by the same fundamental rules. To support this idea, we mention a recent work on a polymeric liquid under $1 \mathrm{D}$ and $2 \mathrm{D}$ nanoconfinement where the authors shows that the exponent for the density scaling of the segmental relaxation time is not affected by the confinement dimensionality and size and agrees with the value from bulk measurements [15].

Motivated by these results, in the present work we study, via Molecular Dynamics (MD) simulations in two dimensions (2D), the impact of the reduction in spatial dimensionality on the scaling between fast dynamics and relaxation. Here we focus on a model of liquid metal, $\mathrm{Cu}_{50} \mathrm{Zr}_{50}$, which is known to have the best glass-forming ability among binary metallic systems in three dimensions (3D) [16]. In agreement with previous works [17, 18], we find that both fast dynamics localization and structural relaxation are affected by long-wavelength fluctuations specific to low-dimensional systems. If these effects are properly treated, the scaling between $\left\langle u^{2}\right\rangle$ and $\tau_{\alpha}$ is recovered which exhibit the same master curve observed in 3D bulk with no adjustable parameters.

The paper is organized as follows. In Sec. 2 details about the numerical models are given. The results are presented and discussed in Sec. 3 and the main conclusions are summarized in Sec. 4. 


\section{Methods}

MD simulations for the CuZr binary alloy were carried out in 2D using LAMMPS molecular dynamics software [19]. An embedded atom model (EAM) potential was used to describe the interatomic interactions [20]. Each simulation consists of a total number of $N=3200$ atoms contained in a box with periodic boundary conditions. The equations of motion are integrated using the velocity Verlet algorithm with a time $\delta t$ which ranges in $10^{-4}-2 \times 10^{-3} \mathrm{ps}$. The temperature $T$ is defined from the kinetic energy, $T=\sum_{i}^{N} m_{i}\left|\mathbf{v}_{\mathbf{i}}\right|^{2} / 2 N k_{B}$, where $\mathbf{v}_{\mathbf{i}}$ and $m_{i}$ are the velocity and mass of the $i$ th atom and $k_{B}$ is the Boltzmann constant. The pressure $\mathrm{P}$ is defined via the virial, $P=\rho k_{B} T+1 / 2 V\left\langle\sum_{i<j} \mathbf{f}_{\mathbf{i j}} \cdot \mathbf{r}_{\mathbf{i j}}\right\rangle$, where $\rho$ is the number density, $V$ the volume and $\mathbf{f}_{\mathbf{i j}}$ is the force between atoms $i$ and $j$ at distance $\mathbf{r}_{\mathbf{i j}}$.

Supercooled samples at different temperatures were generated with the following protocol. Initial configurations were equilibrated at $2000 \mathrm{~K}$, rapidly quenched directly at the target temperature and then equilibrated in the NPT ensemble at zero pressure for a time sufficient to remove all the transient effects in energy and dynamics (order of $100 \tau_{\alpha}$ ). Production runs were carried out in the NVE ensemble, which extend in time until the onset of diffusive regime. With the same protocol, we also carried out simulations under positive external pressure, $P=5 \mathrm{GPa}$ and $P=10 \mathrm{GPa}$ (if not mentioned, in the following discussion data refers to $P=0$ conditions). Ten to twenty independent realizations of each state were considered to ensure suitable statistical average.

\section{Results and discussion}

\subsection{Structural characterization of the supercooled state}

First, we study the evolution of the liquid structure in the supercooled state as denoted by global radial distribution function $g(r)$ and the static structure factor $S(q)$, defined as:

$$
\begin{aligned}
& g(r)=\frac{1}{2 \pi r \Delta r \rho(N-1)}\left\langle\sum_{j \neq i} \delta\left(\left|\mathbf{r}_{i j}\right|-r\right)\right\rangle \\
& S(q)=\frac{1}{N}\left\langle\sum_{i} \sum_{j} e^{-i \mathbf{q}\left(\mathbf{r}_{j}-\mathbf{r}_{i}\right)}\right\rangle
\end{aligned}
$$

where $\mathbf{r}_{i}$ is the position of the $i$ th atom, $\mathbf{r}_{i j}=\mathbf{r}_{j}-\mathbf{r}_{i}$ and $\langle\ldots\rangle$ denotes the ensemble average. In Figure $1 g(r)$ and $S(q)$ are shown for selected temperatures. The lack of longrange translational order, typical of the supercooled liquid state, is apparent in both the quantities. On the other hand, the splitting of the second peak developing upon cooling could be the signature of the emergence of medium-range ordering. For the present model, $\mathrm{Hu}$ et al. demonstrated that in 2D growing local crystal-like order controls the slowing-down of dynamics in the supercooled liquid and play a crucial role in the formation of a 2D metallic glass. [21]. 

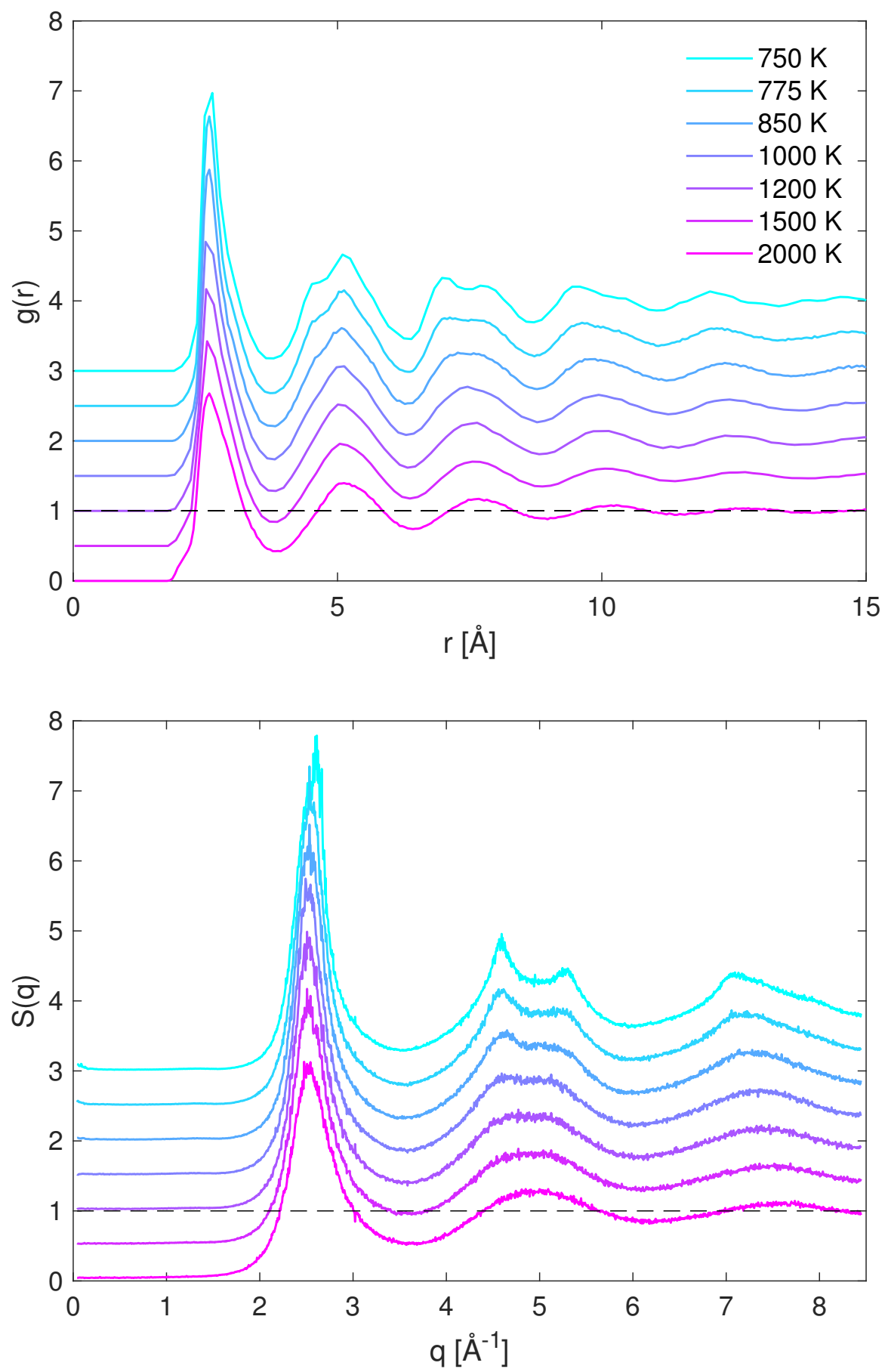

Figure 1. Radial distribution functions $\mathrm{g}(\mathrm{r})$ (top panel) and structure factors $\mathrm{S}(\mathrm{q})$ (bottom panel) at selected temperatures. The data have been shifted for clarity. 


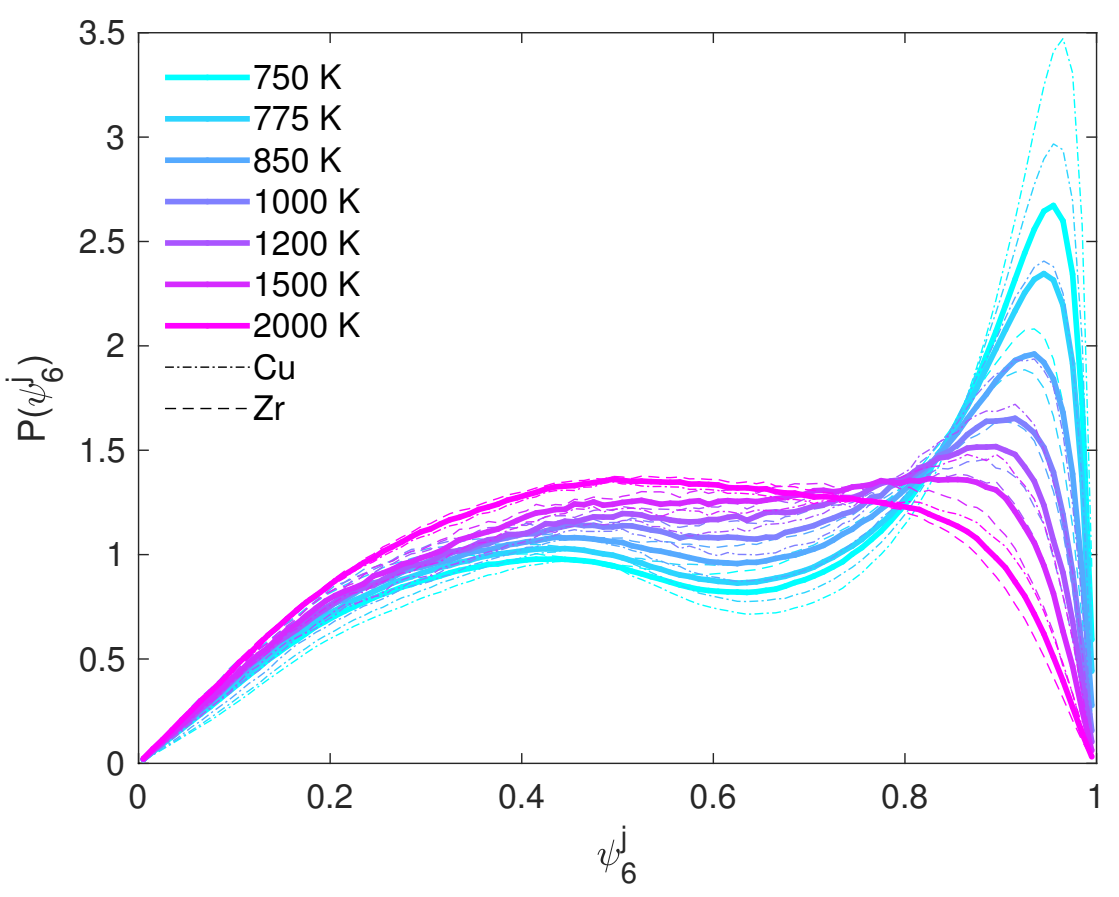

Figure 2. Distribution $P\left(\psi_{6}^{j}\right)$ of the hexatic-order parameter $\psi_{6}^{j}$ for $\mathrm{Cu}$ atoms (dash-dotted lines), $\mathrm{Zr}$ atoms (dashed lines) and global (solid lines), at selected temperatures.

In order to deepen the structural aspects, we consider the hexatic-order parameter $\psi_{6}^{j}$ :

$$
\psi_{6}^{j}=\frac{1}{n_{j}} \sum_{m=1}^{n_{j}}\left|e^{i 6 \theta\left(\mathbf{r}_{m j}\right)}\right|
$$

where $\theta\left(\mathbf{r}_{m j}\right)$ is the angle between $\mathbf{r}_{m j}=\mathbf{r}_{m}-\mathbf{r}_{j}$ and the $\mathrm{x}$ axis, $n_{j}$ is the nearest neighbors number of $j$ th atom. Figure 2 displays the temperature dependence of the $\psi_{6}^{j}$ distribution $P\left(\psi_{6}^{j}\right)$ for $\mathrm{Cu}$ and $\mathrm{Zr}$ atoms. At high temperature $\psi_{6}^{j}$ is broadly distributed and $P\left(\psi_{6}^{j}\right)$ does not exhibit any peculiar feature. At lower temperature, a peak appears for large $\psi_{6}^{j}$ values, corresponding to high local bond orientational order (BOO), that becomes more pronounced at deeper supercooling. The distribution becomes bimodal, with a minimum occurring at $\psi_{6}^{*}=0.645$. This signals the presence of structural heterogeneities, in agreement with the results of $\mathrm{Hu}$ and coworkers [21]. This tendency of increasing BOO is also clear in Figure 3 where we compare two snapshots of the spatial distribution of $\psi_{6}^{j}$ at high (top) and low (bottom) temperature. At low $\mathrm{T}$ we observe some spatially extending regions of high BOO which are not detected at high temperature. This regions should not be confused with crystal nuclei developing in a crystallizing system as the former are characterized by a finite lifetime and their contribution does not increase in time, i.e., the corresponding distribution $P\left(\psi_{6}^{j}\right)$ in Figure 2 is stationary. 

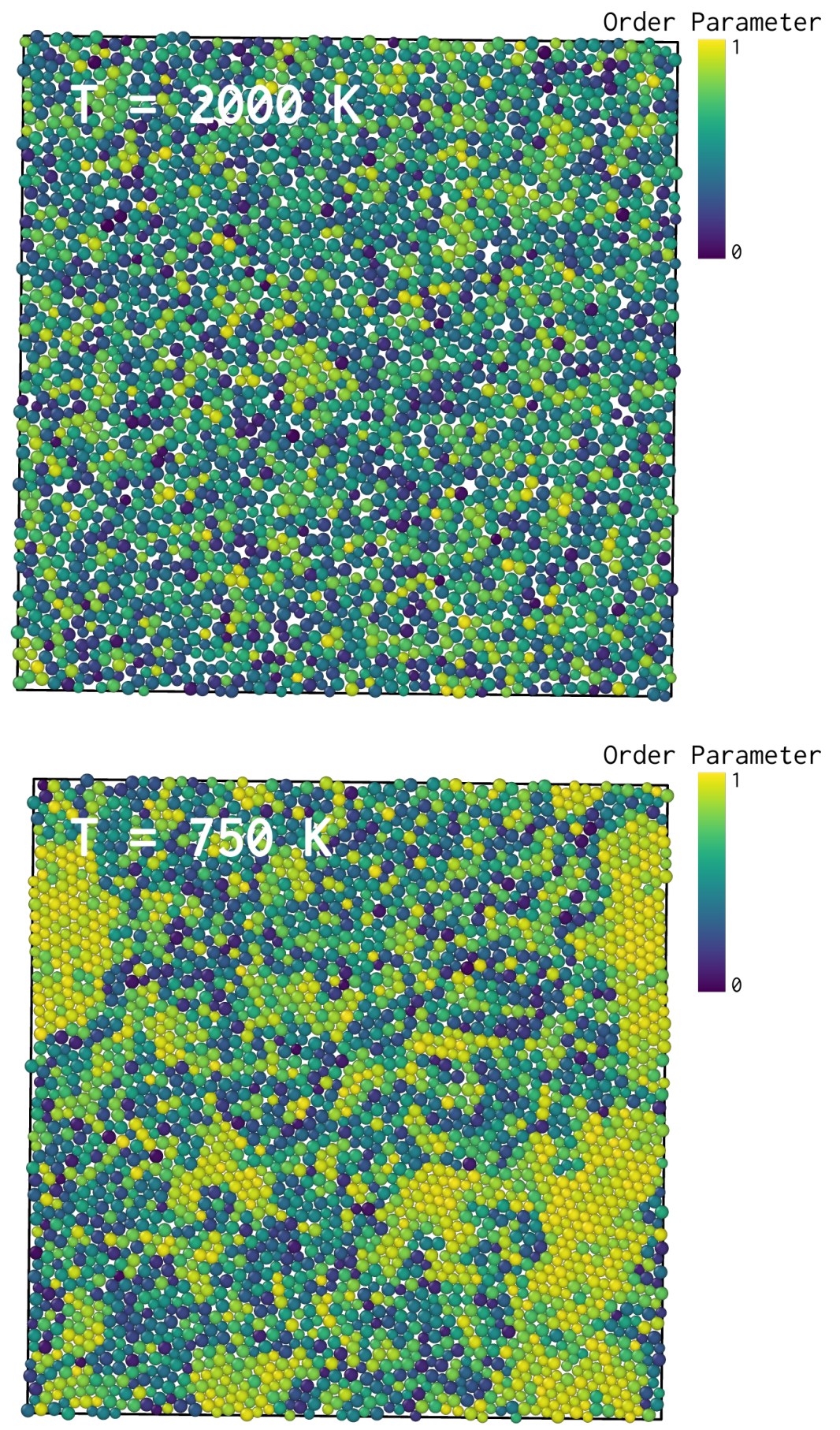

Figure 3. Representative spatial distribution of the hexatic-order parameter $\psi_{6}^{j}$ for $T=$ $2000 \mathrm{~K}$ (top) and $T=750 \mathrm{~K}$ (bottom). 


\subsection{Slowing-down of dynamics: localization and structural relaxation}

In this section we investigate the slowing-down of dynamics in the supercooling regime, focusing on the two aspects, particle localization and structural relaxation. Atomic mobility is evaluated via the mean square displacement (MSD):

$$
\left\langle\Delta r^{2}(t)\right\rangle=\frac{1}{N}\left\langle\sum_{j=1}^{N}\left\|\mathbf{r}_{j}(t)-\mathbf{r}_{j}(0)\right\|^{2}\right\rangle
$$

The structural relaxation is conveniently described by the self part of the intermediate scattering function (ISF):

$$
F_{s}(q, t)=\frac{1}{N}\left\langle\sum_{j=1}^{N} e^{i \mathbf{q} \cdot\left(\mathbf{r}_{j}(t)-\mathbf{r}_{j}(0)\right)}\right\rangle
$$

The structural relaxation time $\tau_{\alpha}$ is defined via $F_{s}\left(q_{\max }, \tau_{\alpha}\right)=1 / e$ where $q_{\max }$ is $q$-vector of the maximum of the static structure factor corresponding to about the distance of nearestneighbors.

In Figure 4 we plot for selected temperature the MSD (panels A and B) and ISF (panels $\mathrm{C}$ and $\mathrm{D}$ ) of $\mathrm{Cu}$ and $\mathrm{Zr}$ atoms. We note that the MSD and ISF curves manifest the features which are peculiar of glassy dynamics in 2D and mark the difference with the 3D analogue. Particle localization corresponding to the cage effect, apparent in 3D at low temperature, is virtually absent in 2D. Indeed, the intermediate time plateau is not observed in the ISF and is replaced by a sub-diffusive regime in the MSD. Structural relaxation is also different in 2D and 3D: the quasi-exponential decay of the ISF in 3D is replaced by an extremely slow decay in 2D in which the noise level seems amplified even though the average over 20 independent realizations was considered to improve the statistical precision. These observations agree with previous findings in simulations of binary disks interacting via a Lennard-Jones [17] or soft-core potential [22] as well as experiments on quasi-2D colloids [18] and hard-spheres [23].

For sake of completeness, we consider the non-gaussian parameter (NGP)[24]:

$$
\alpha_{2}(t)=\frac{\left\langle\Delta r^{4}(t)\right\rangle}{(1+2 / d)\left\langle\Delta r^{2}(t)\right\rangle^{2}}-1
$$

where $\left\langle\Delta r^{4}(t)\right\rangle$ is the mean fourth displacement and $d$ the spatial dimension. The NGP quantifies the deviations from the gaussian character of the displacement ascribed to dynamic heterogeneities (DHs). The insets of panel A and B in Fig.4 display the NGP of Cu and Zr atoms. We note that, despite the low temperature values attained, the peak value of the NGP, $\alpha_{2, \max }$, remains rather low, $\alpha_{2, \max }<1$, indicating a modest degree of heterogeneity.

It is now well established that long-wavelength fluctuations, known as Mermin-Wagner fluctuations, are responsible for the vanishing of the transient solid-like dynamics in the supercooled regime [25]. To disentangle the influence of these fluctuations on glassy dynamics, it has been proposed to consider cage-relative (CR) particle motion, namely to measure for each atom the displacement relative to the cage of its neighbors. In this spirit, we define a CR particle displacement:

$$
\Delta \mathbf{r}_{\mathrm{CR}, j}(t)=\mathbf{r}_{j}(t)-\mathbf{r}_{j}(0)-\Delta \mathbf{r}_{\mathrm{C}, j}(t)
$$



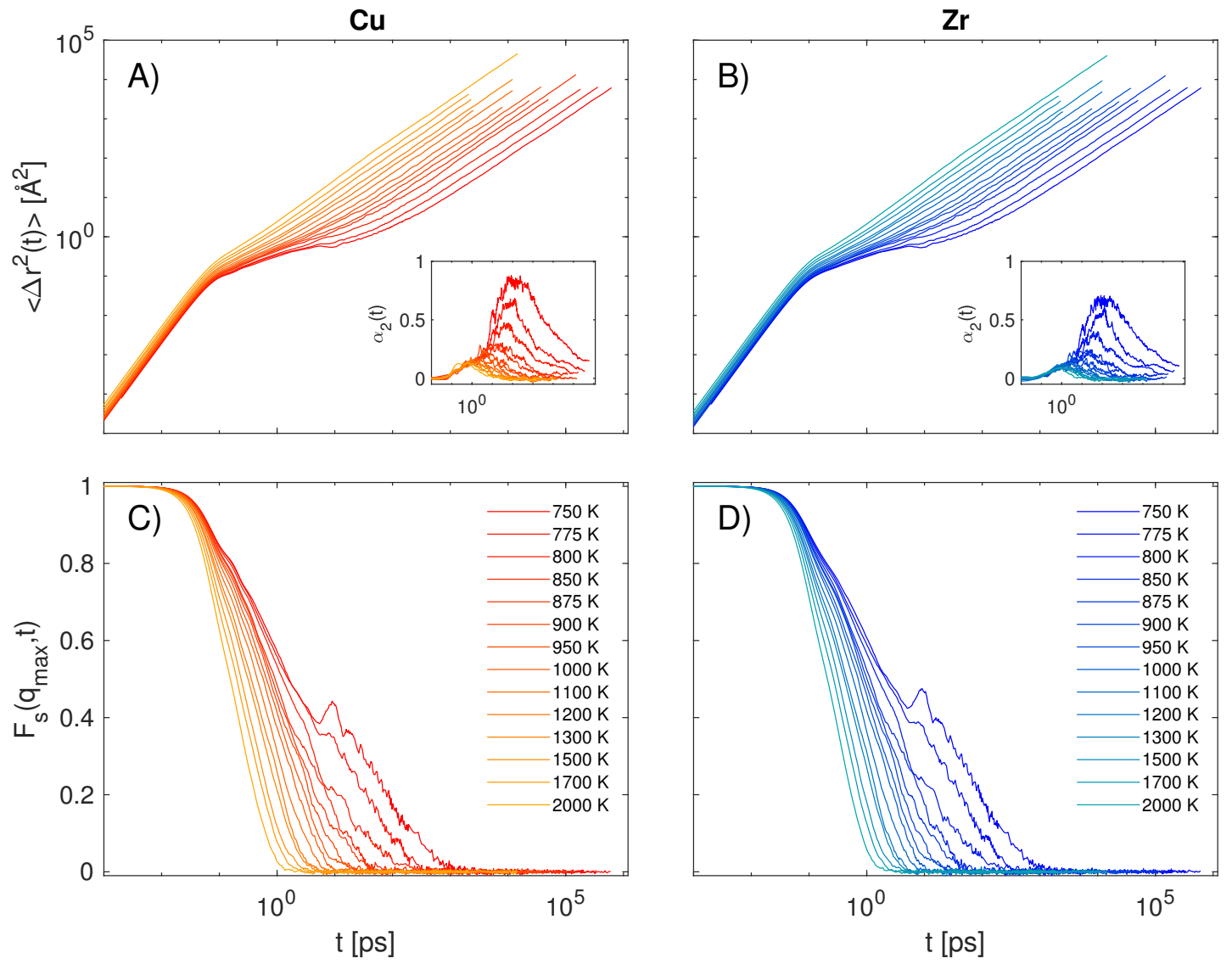

Figure 4. Panels $\mathrm{A}$ and $\mathrm{B}$ : mean square displacement $\left\langle\Delta r^{2}(t)\right\rangle$ of $\mathrm{Cu}(\mathrm{A})$ and $\mathrm{Zr}(\mathrm{B})$ atoms at selected temperatures. Insets: corresponding non-gaussian parameters $\alpha_{2}(t)$. Panels $\mathrm{C}$ and D: corresponding self part of the intermediate scattering function $F_{s}(q, t)$ of $\mathrm{Cu}(\mathrm{C})$ and $\mathrm{Zr}(\mathrm{D})$. The $q$-vector $q_{\max }$ corresponding to the maximum of the static structure factor is considered.

where

$$
\Delta \mathbf{r}_{\mathrm{C}, j}(t)=\frac{1}{N_{j}} \sum_{i=1}^{N_{j}}\left(\mathbf{r}_{i}(t)-\mathbf{r}_{i}(0)\right)
$$

The sum in Eq. 8 runs over all the nearest neighbors of the $j$ th atom, i.e. the atoms whose distance from the central one at time 0 is smaller than the value corresponding to the first minimum in the $g(r)$. Then, we introduce a CR mean square displacement (CR-MSD):

$$
\left\langle\Delta r_{\mathrm{CR}}^{2}(t)\right\rangle=\frac{1}{N}\left\langle\sum_{j=1}^{N}\left\|\left(\mathbf{r}_{j}(t)-\mathbf{r}_{j}(0)\right)-\Delta \mathbf{r}_{\mathrm{C}, j}(t)\right\|^{2}\right\rangle
$$

and a CR intermediate scattering function (CR-ISF):

$$
F_{s, \mathrm{CR}}(q, t)=\frac{1}{N}\left\langle\sum_{j=1}^{N} e^{i \mathbf{q} \cdot\left(\mathbf{r}_{j}(t)-\mathbf{r}_{j}(0)-\Delta \mathbf{r}_{\mathrm{C}, j}(t)\right)}\right\rangle
$$

A CR non-gaussian parameter (CR-NGP) can also be defined by replacing in Eq. $6\left\langle\Delta r^{2}(t)\right\rangle$ and $\left\langle\Delta r^{4}(t)\right\rangle$ with the corresponding CR quantities $\left\langle\Delta r_{\mathrm{CR}}^{2}(t)\right\rangle$ and $\left\langle\Delta r_{\mathrm{CR}}^{4}(t)\right\rangle$. 

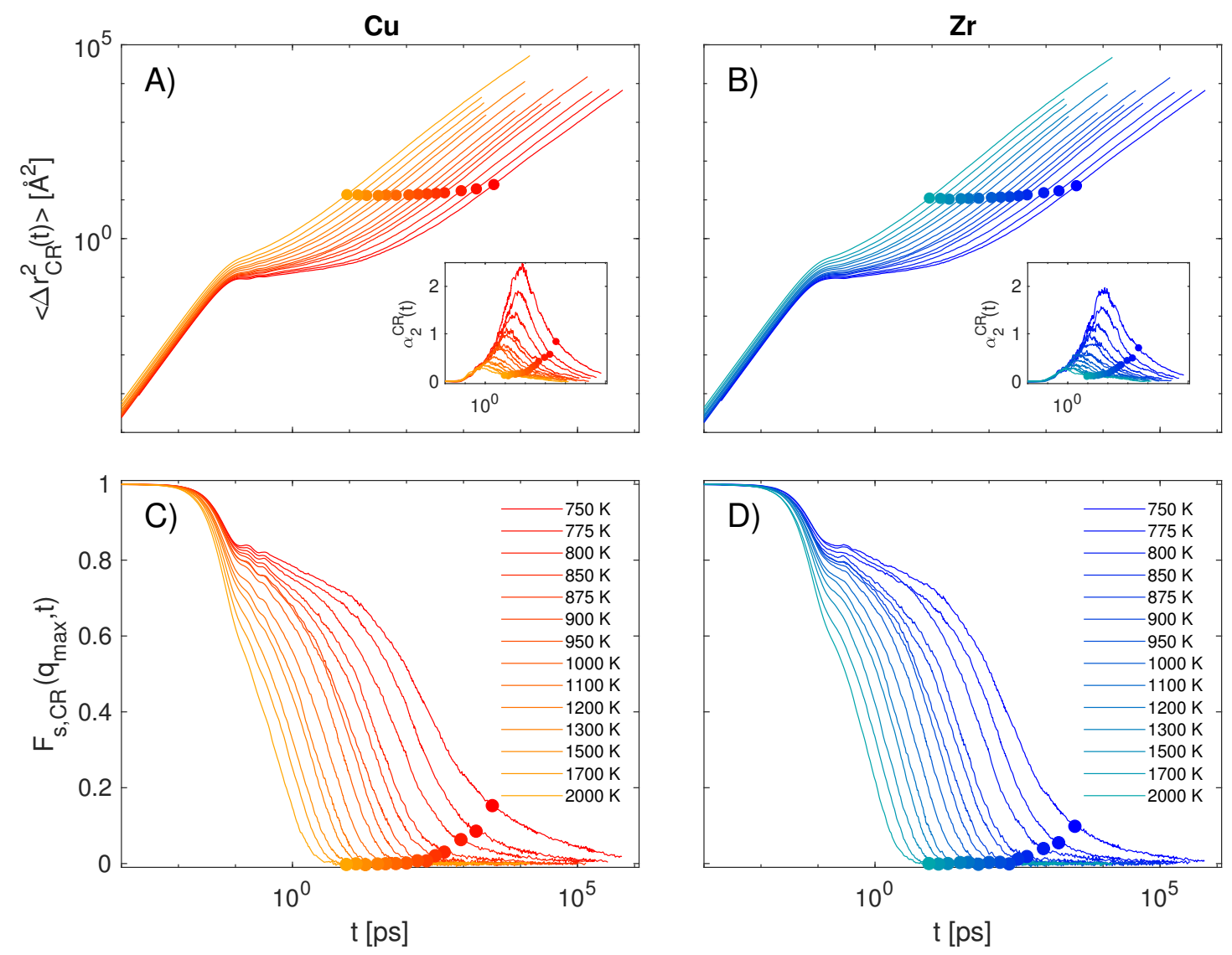

Figure 5. Panels $\mathrm{A}$ and $\mathrm{B}$ : cage-relative mean square displacement $\left\langle\Delta r_{\mathrm{CR}}^{2}(t)\right\rangle$ of $\mathrm{Cu}$ (A) and $\mathrm{Zr}(\mathrm{B})$ atoms at selected temperatures. Insets: corresponding cage-relative non-gaussian parameters $\alpha_{2}^{\mathrm{CR}}(t)$. Panels C and D: corresponding cage-relative self part of the intermediate scattering function $F_{s, \mathrm{CR}}(q, t)$ of $\mathrm{Cu}(\mathrm{C})$ and $\mathrm{Zr}(\mathrm{D})$. The $q$-vector $q_{\max }$ corresponding to the maximum of the static structure factor is considered. Full circles mark the cage lifetime $\tau_{c}$ (see the text for more details).

In Figure 5 we show the CR-MSD (panels $\mathrm{A}$ and $\mathrm{B}$ ), the CR-ISF (panels $\mathrm{C}$ and $\mathrm{D}$ ) and CR-NGP (insets of panels $\mathrm{A}$ and $\mathrm{B}$ ) of $\mathrm{Cu}$ and $\mathrm{Zr}$ atoms for selected temperatures (for comparison, the corresponding standard MSD, ISF and NGP curves are shown in Figure 4). As expected, if we assume the CR perspective, the dynamical signature of the supercooled state, in particular localization and structural relaxation, emerge and it is virtually impossible to distinguish the 2D system from its 3D counterpart only by visual inspection (see [16] for the data of the 3D system).

A crucial aspect of the CR approach is how far in time one can push its validity. Indeed, we expect that, after a given time, due to cage breaking and diffusive processes, one atom will necessarily have lost those neighbors which were detected at time 0 . To deepen this aspect, we consider for a given atom the fraction $\phi(t)$ of its original neighbors which are still present in the cage after a time $t$. We define a cage lifetime $\tau_{c}$ as $\phi\left(\tau_{c}\right)=\left(\bar{n}_{\mathrm{NN}}-4\right) / \bar{n}_{\mathrm{NN}}$ where $\bar{n}_{\mathrm{NN}} \approx 7$ is the mean number of nearest neighbors. This criterion set the vanishing of the cage when at least 4 initial neighbors are lost, building on the observation that this is the minimum 
value for the neighbor exchange in order to have an irreversible cage breaking [26]. If we mark the cage lifetime $\tau_{c}$ on the CR-MSD and CR-ISF curves in Figure 5, we observe that it occurs when the final decay of the CR-ISF, denoting structural relaxation, is completed.

Being the purpose of the present work to discuss the connection between fast dynamics within the cage and structural relaxation, in the following we will consider mainly cage relative dynamical properties. Hence, we define the cage relative Debye-Waller (CR-DW) factor $\left\langle u_{\mathrm{CR}}^{2}\right\rangle=\left\langle\Delta r_{\mathrm{CR}}^{2}\left(t_{D W}\right)\right\rangle$ where $t_{D W}$ is a measure of the trapping time of a particle in the cage $[1,4]$. In agreement with the corresponding $3 \mathrm{D}$ model, we set $t_{D W}=1 \mathrm{ps}$ as it ensures that, at sufficiently low temperature, $\left\langle u_{\mathrm{CR}}^{2}\right\rangle$ corresponds to the plateau value of the mean square displacement denoting particle caging. Similarly, the cage relative structural relaxation time $\tau_{\alpha}^{\mathrm{CR}}$ is defined via $F_{s, \mathrm{CR}}\left(q_{\max }, \tau_{\alpha}^{\mathrm{CR}}\right)=1 / e$.

Figure 6 shows the temperature dependence of $\left\langle u_{\mathrm{CR}}^{2}\right\rangle$ (panel A) and $\tau_{\alpha}^{\mathrm{CR}}$ (panel B) for $\mathrm{Cu}$ and $\mathrm{Zr}$ atoms. We observe that the CR-DW extrapolates to zero at a finite temperature, $T_{0, D W} \approx 510 \mathrm{~K}$ for $\mathrm{Cu}$ and $T_{0, D W} \approx 470 \mathrm{~K}$ for $\mathrm{Zr}$, in agreement with previous results in 3D metallic systems $[27,16]$ and polymers [28]. Further, in the supercooled regime the behavior of the CR structural relaxation time can be described by the Vogel-Fulcher-Tamman (VFT) equation, $\tau_{\alpha}^{\mathrm{CR}}=\tau_{0} \exp \left[B T_{0} /\left(T-T_{0}\right)\right]$. The fitted values of $T_{0}, T_{0} \approx 565 \mathrm{~K}$ and $T_{0} \approx 550 \mathrm{~K}$ for $\mathrm{Cu}$ and $\mathrm{Zr}$ atoms respectively, with their uncertainty, compares well with the values of $T_{0, \mathrm{DW}}$ from the CR-DW factor.

\subsection{Scaling between fast dynamics and relaxation}

Extensive simulation works and experiments revealed strong correlations between fast vibrational dynamics and structural relaxation, which can be expressed as a master curve $\tau_{\alpha}=\mathcal{F}\left(\left\langle u^{2}\right\rangle\right)$, connecting the Debye-Waller factor $\left\langle u^{2}\right\rangle$ and the structural relaxation time $\tau_{\alpha}$. Here, we consider the master curve proposed by Larini et al. [1]:

$$
\tau_{\alpha}=\tau_{0} \exp \left(\frac{\overline{a^{2}}}{2\left\langle u^{2}\right\rangle}+\frac{\sigma_{a^{2}}^{2}}{8\left\langle u^{2}\right\rangle^{2}}\right)
$$

where $\tau_{0}, \overline{a^{2}}$ and $\sigma_{a^{2}}^{2}$ are system-dependent parameters. Eq.11 improves the relation originally proposed by Hall and Wolynes [29] and accounts for the convexity of the master curve, evidenced by experiments and simulations. Eq.11 is recast in the universal form:

$$
\tau_{\alpha}=\tilde{\tau}_{0} \exp \left[\tilde{\beta}\left(u_{g}^{2} /\left\langle u^{2}\right\rangle\right)+\tilde{\gamma}\left(u_{g}^{2} /\left\langle u^{2}\right\rangle\right)^{2}\right]
$$

being $u_{g}^{2}$ the DW factor at GT (defined via $\tau_{\alpha}=10^{2} \mathrm{~s}$ or $\eta=10^{12} \mathrm{~Pa} \cdot \mathrm{s}$ ) [1]. In particular, now the universal constants $\hat{\beta}=\tilde{\beta} \ln 10=3.7(1)$ and $\hat{\gamma}=\tilde{\gamma} \ln 10=28.4(2)$ are introduced, with $\tilde{\beta}$ and $\tilde{\gamma}$ defined in [1], and $\tau_{0}$ ensures $\tau_{\alpha}=10^{2}$ s at GT [1]. Indeed, Eq. 12 was shown to provide a good description of experimental data in several systems $[1,9,4]$.

Figure 7 is a correlation plot between the structural relaxation time and the inverse DW factor, both quantities derived from cage-relative dynamics, for $\mathrm{Cu}$ and $\mathrm{Zr}$ components. To test the robustness of this correlation, in addition to the simulation data at zero pressure discussed above, in Fig. 7 we also include data obtained imposing an external pressure on 

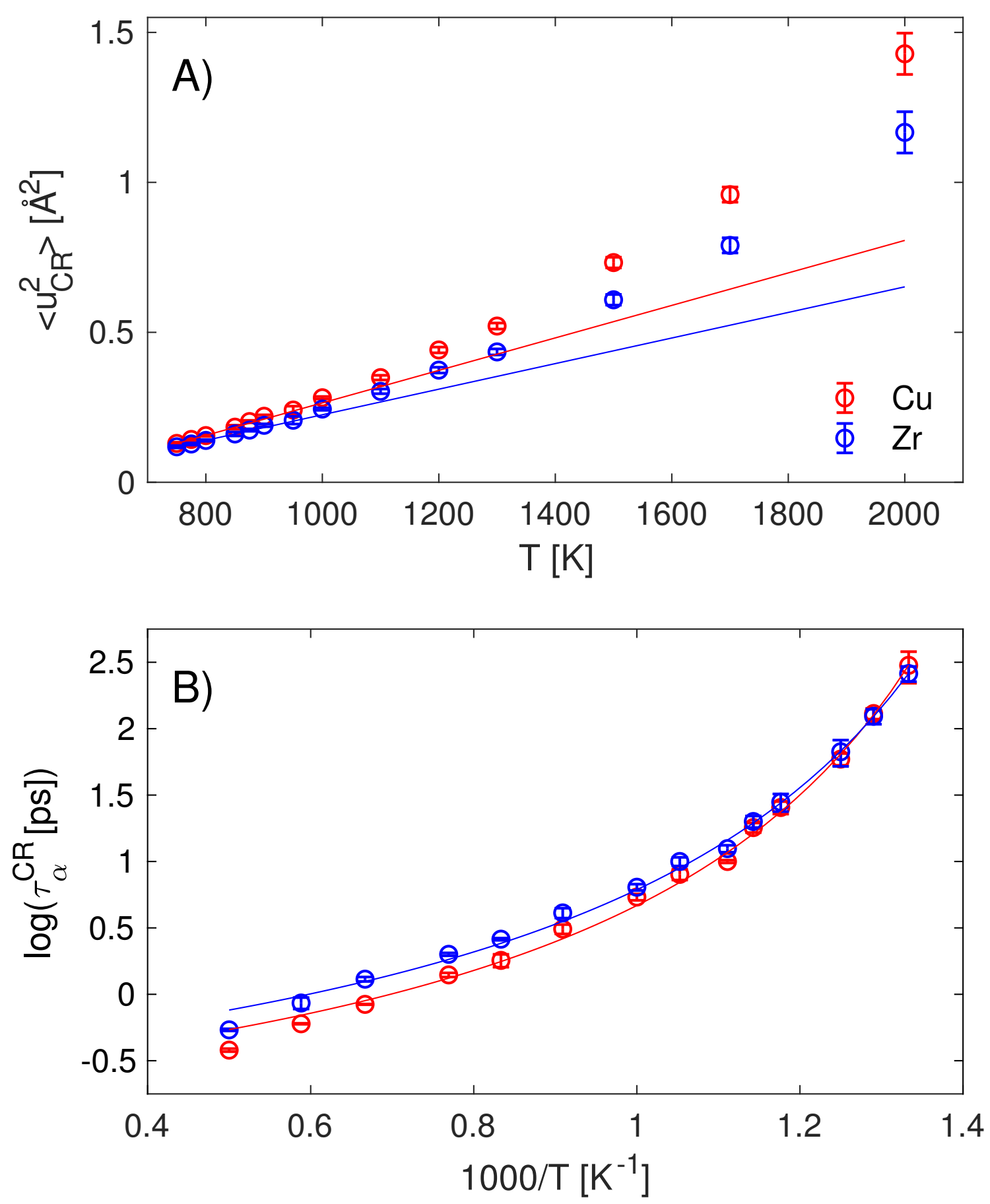

Figure 6. Panel A: temperature dependence of CR-DW factor $\left\langle u_{\mathrm{CR}}^{2}\right\rangle$ for $\mathrm{Cu}$ and $\mathrm{Zr}$ atoms. The full lines correspond to the linear fit in the low temperature range. Panel B: Arrhenius plot of the $\mathrm{CR}$ structural relaxation time $\tau_{\alpha}^{\mathrm{CR}}$ for $\mathrm{Cu}$ and $\mathrm{Zr}$ atoms. Full lines are fitted with the Vogel-Fulcher-Tammann (VFT) law $\tau_{\alpha}^{\mathrm{CR}}=\tau_{0} \exp \left[B T_{0} /\left(T-T_{0}\right)\right]$. 


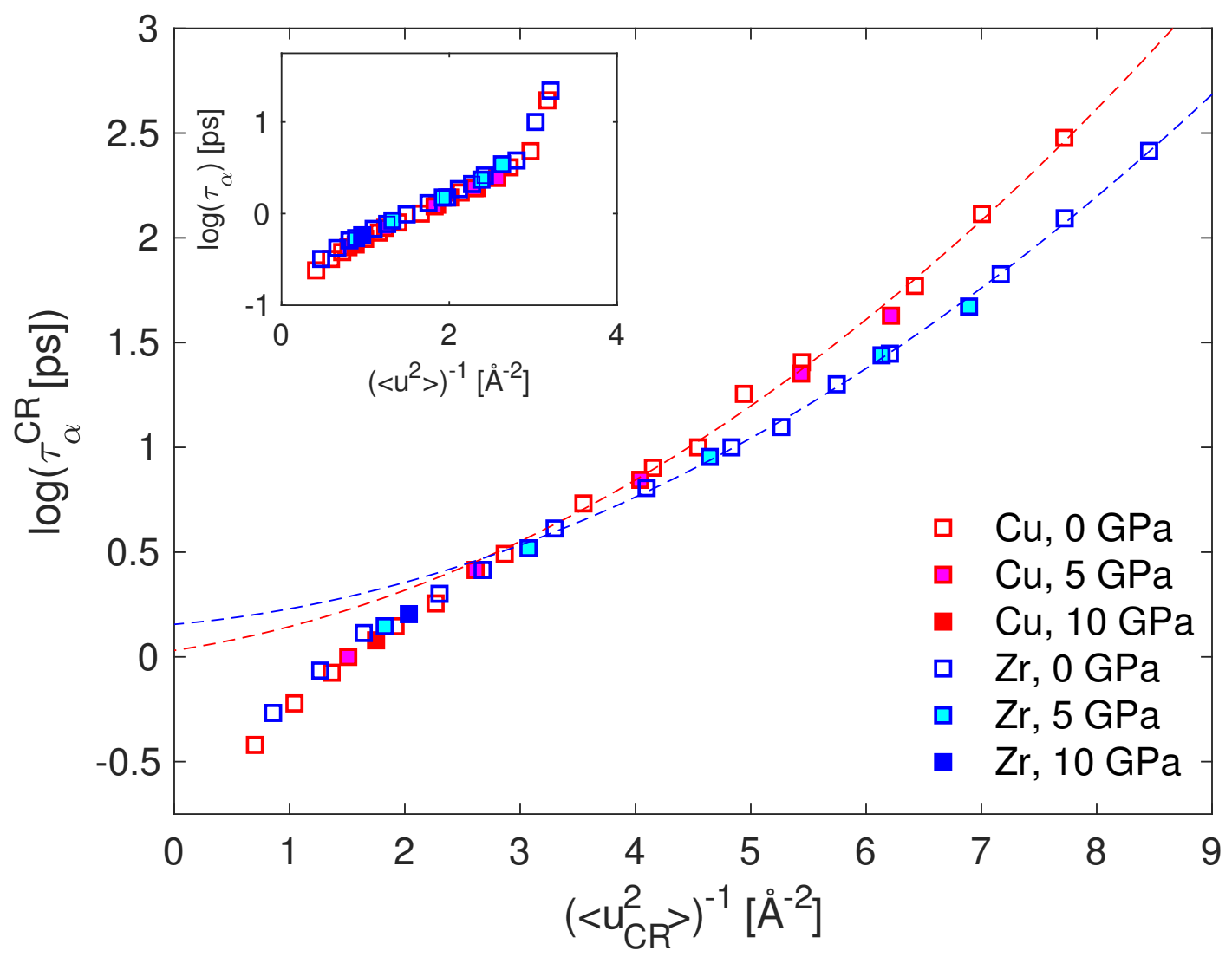

Figure 7. Main panel: correlation plot of the CR structural relaxation time $\tau_{\alpha}^{\mathrm{CR}}$ and CR-DW factor $\left\langle u_{\mathrm{CR}}^{2}\right\rangle$ of $\mathrm{Cu}$ and $\mathrm{Zr}$ atoms. Data for different pressure conditions are shown. Dashed lines are fitted with the master curve defined in Eq. 11. Inset: corresponding correlation plot of the usual structural relaxation time $\tau_{\alpha}$ and DW factor $\left\langle u^{2}\right\rangle$ (see the text for more details).

the system. The superimposed curves are the best-fit with Eq. 11, restricted to those states for which $\log \tau_{\alpha}^{\mathrm{CR}} \geq 0.5$, i.e., structural relaxation is reasonably separated from the fast dynamics timescale. We note that Eq. 11 satisfactorily account for the correlation between $\tau_{\alpha}^{\mathrm{CR}}$ and $\left\langle u_{\mathrm{CR}}^{2}\right\rangle$.

Douglas and coworkers [3], building on free-volume arguments, developed a localization model predicting an alternative master curve $\log \tau_{\alpha} \propto\left\langle u^{2}\right\rangle^{-\alpha / 2}$ where the exponent $\alpha$ would equal the spatial dimensionality for regular volume shapes, like a sphere in 3D or a circle in 2D. Then, according to this picture, the dependence of $\tau_{\alpha}$ on $\left\langle u^{2}\right\rangle$ is different in 2D and 3D. Our results seem not to support this scenario, as we have shown that a 2D system obeys the same scaling law which was derived for 3D systems.

The inset of Fig. 7 presents an alternative analysis of the correlation, in which the usual DW factor $\left\langle u^{2}\right\rangle$ and structural relaxation time $\tau_{\alpha}$ are examined. The standard scaling is significantly different from the CR one, being all the data compressed in the short $\tau_{\alpha}$ and large $\left\langle u^{2}\right\rangle$ region. Anyway, this approach is at least questionable since, as we discussed above, these quantities are not good estimates of particle localization or structural relaxation. 


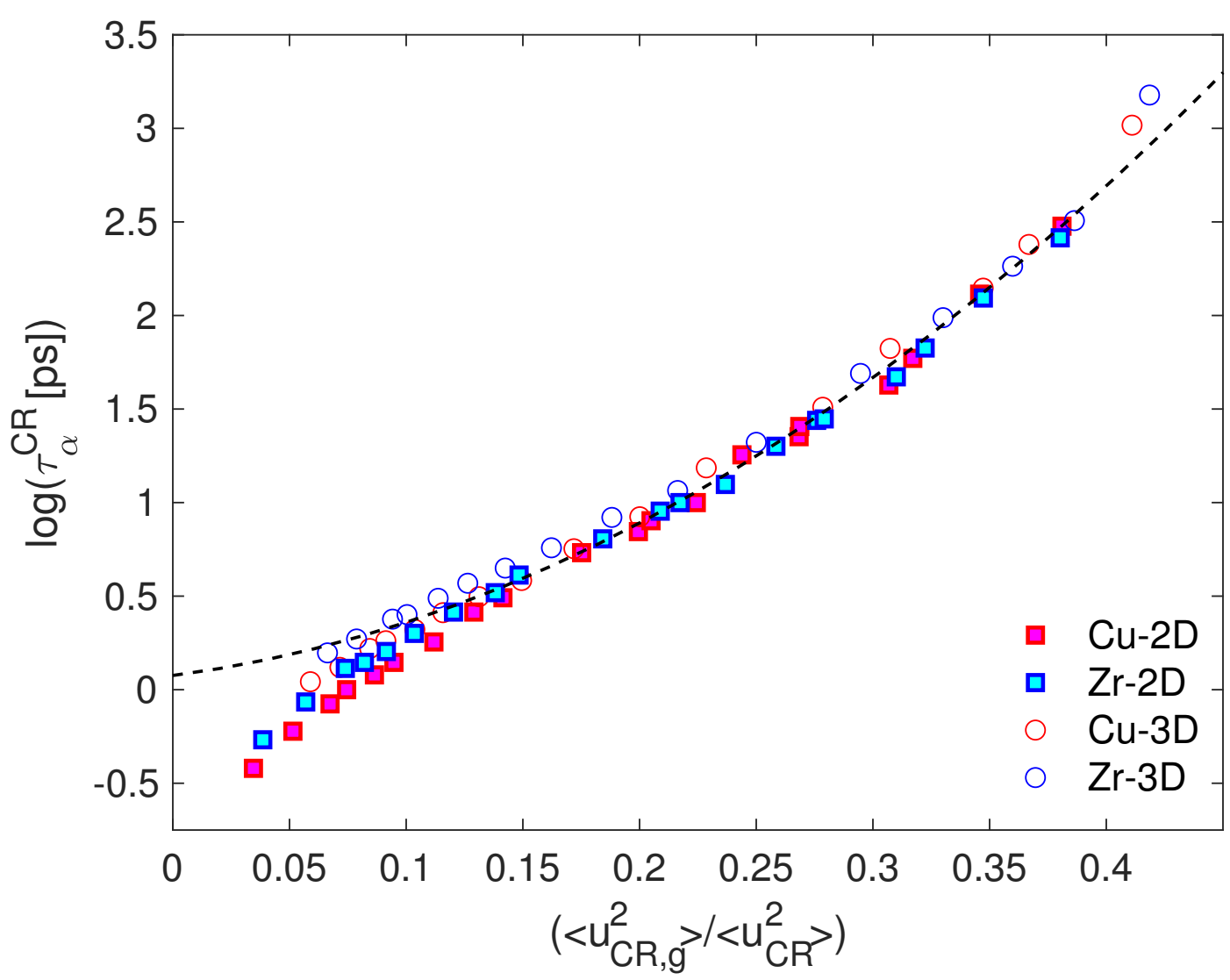

Figure 8. Universal scaling between the structural relaxation time and the DW factor. Data for the 2D and 3D models of the metallic glass-forming liquid are shown. The dashed line is the universal master curve, defined in Eq. 12.

In Figure 8 we plot the $\mathrm{CR}$ structural relaxation time as a function of the rescaled variable $\left\langle u_{\mathrm{CR}, g}^{2}\right\rangle /\left\langle u_{\mathrm{CR}}^{2}\right\rangle$ where $\left\langle u_{\mathrm{CR}, g}^{2}\right\rangle$ is the value of the CR-DW factor at the glass transition. Following previous approach [1], we estimate the value of $\left\langle u_{\mathrm{CR}, g}^{2}\right\rangle$ by extrapolating Eq. 11; this yields $\left\langle u_{\mathrm{CR}, g}^{2}\right\rangle=0.049 \AA^{2}$ for $\mathrm{Cu}$ and $\left\langle u_{\mathrm{CR}, g}^{2}\right\rangle=0.045 \AA^{2}$ for Zr. We complement the results from the 2D model of metallic liquid with the data for equivalent 3D model, taken from [8]. Simulation data are compared with Eq. 12 with no adjustment.

Figure 8 is the major result of the present work. The collapse of the data evidences that structural relaxation and vibrational dynamics of the 2D metallic liquid exhibit the same scaling of 3D systems. The scaling form given by Eq. 12, originally found in bulk and confined 3D systems, also works as a master curve in 2D.

\subsection{Density scaling}

Finally, we discuss the correlation between fast dynamics and relaxation within the framework of the so-called density scaling. In the past decades, following the advent of high-pressure experiments, a remarkable result in understanding the relaxation and the transport of glass- 

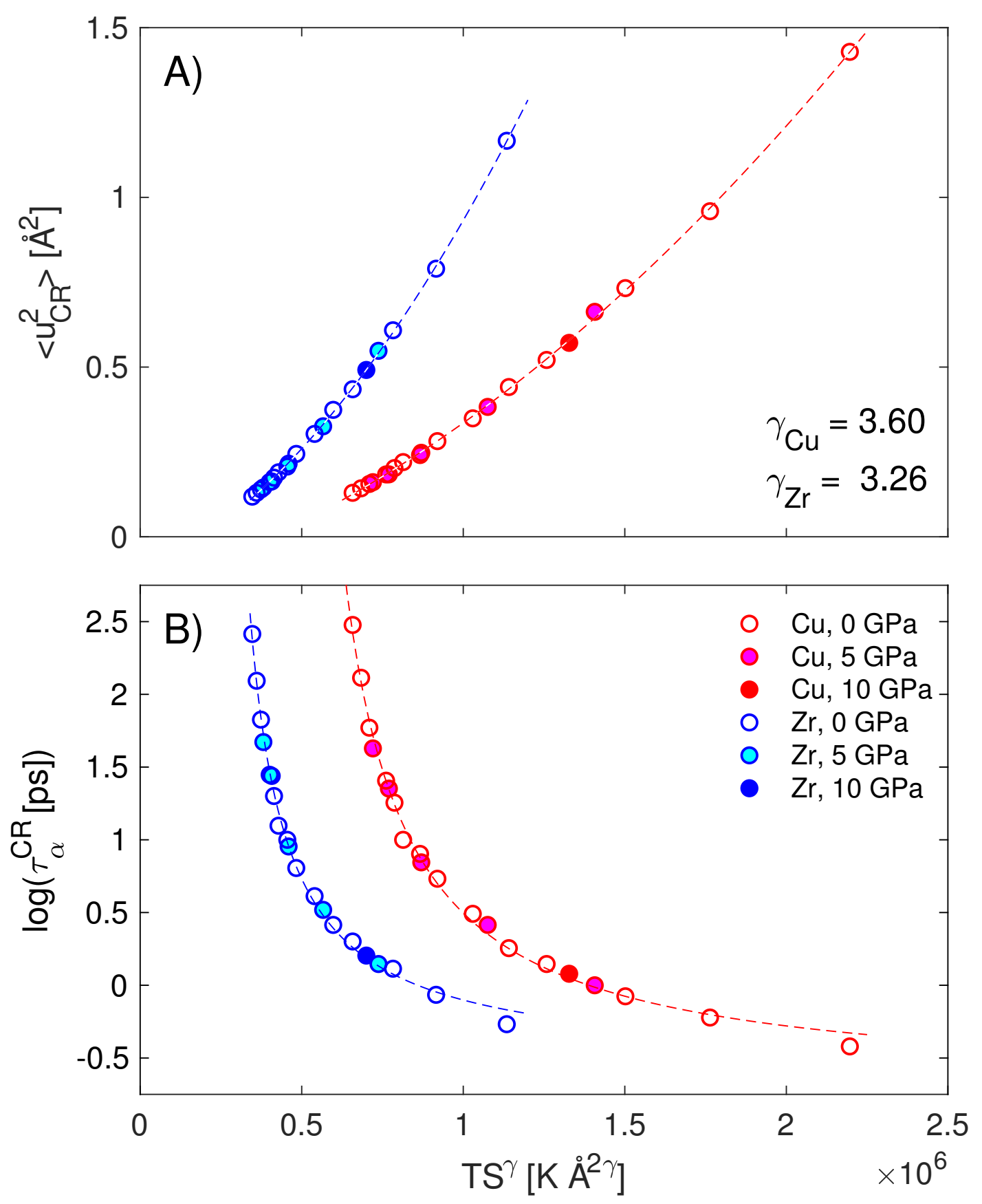

Figure 9. Density scaling of the CR-DW factor $\left\langle u_{\mathrm{CR}}^{2}\right\rangle$ (panel A) and CR structural relaxation time $\tau_{\alpha}^{\mathrm{CR}}$ (panel B). The values of the scaling exponents are $\gamma_{\mathrm{Cu}}=3.60$ and $\gamma_{\mathrm{Zr}}=3.26$.

forming liquids was the discovery that evolution of the structural relaxation time or the viscosity, measured under different $T$ and $P$ conditions, can be described through a simple scaling relation, $T \rho^{-\gamma}$ or equivalently $T v^{\gamma}$, where $\rho$ and $v$ are the density and specific volume (see [30] for a review on this topic). Both the exponent $\gamma$ and the specific master curve are system-dependent. Even though the density scaling has been mostly investigated on the time scale of the structural relaxation or viscous flow, it was also observed on shorter time scales, 
in particular those pertaining to fast dynamics. Density scaling of the DW factor has been reported by previous MD studies in molten salt CKN [31], polymers [28, 32] and binary atomic mixtures [4].

On this basis, we are interested in testing the density scaling in the present model of $2 \mathrm{D}$ metallic glass-forming liquid. This is done in Figure 9, where we demonstrate the validity of the scaling by plotting the CR-DW factor $\left\langle u_{\mathrm{CR}}^{2}\right\rangle$ (top panel) and the CR structural relaxation time $\tau_{\alpha}^{\mathrm{CR}}$ (bottom panel) as a function of the product $T S^{\gamma}$ (in 2D the volume is replaced by the area $S$ ). We fix the exponent $\gamma$ focusing on the fast dynamics: we assume a polynomial dependence of $\left\langle u_{\mathrm{CR}}^{2}\right\rangle$ on $T S^{\gamma}$ and we determine the value of $\gamma$ for which the coefficient of determination $R^{2}$ of the fit is maximum. Figure 9 indicates that the density scaling of the fast dynamics leads to the scaling of the structural relaxation with the same exponent, suggesting that also in 2D systems the density scaling is rooted in the fast dynamics, in agreement with previous results in 3D [28].

\section{Conclusions}

We studied by MD simulations in two dimensions a model of metallic glass-former, CuZr, which is known to possess the best glass-forming ability in three dimensions. In the supercooled regime, while local crystal-like order develops, long wavelength fluctuations wash out caging effects and glassy structural relaxation, which however are recovered if atomic cage-relative dynamics is considered. We have demonstrated that (i) the structural relaxation time exhibits strong correlation with the fast vibrational dynamics, as accounted for by the DW factor of the atoms, and (ii) the correlation is the same of 3D systems, in the sense that it is described by the same master curve, with no adjustable parameters, originally proposed for 3D bulk materials.

Our results in 2D confirm the robustness of the scaling between fast dynamics and slow relaxation. In particular, they provide the basis for understanding the observations of a bulkequivalent scaling in confined systems [14], as the dynamics within a layer can be considered quasi-2D. Further, Hu et al. in a recent paper showed that for the present model there can be a significant influence of spatial dimensionality on the structural mechanism of glass transition in metallic glasses [21]. Our findings suggest that, despite these structural differences can play a relevant role on both vibrational dynamics and relaxation, they do not affect the deeper aspects of their correlation.

Finally, we note that there is not direct and trivial relation between the present analysis and previous works addressing the effect of spatial dimension in the dynamics of glassforming liquids $[13,14,15]$. Hence, the reported equivalence of $2 \mathrm{D}$ and $3 \mathrm{D}$ scaling is a further indication supporting the idea that the dynamics in bulk and confined glassforming liquids can be described by the same fundamental rules. 


\section{References}

[1] L. Larini, A. Ottochian, C. De Michele, and D. Leporini. Universal scaling between structural relaxation and vibrational dynamics in glass-forming liquids and polymers. Nature Physics, 4:42-45, 2008.

[2] F. Puosi and D. Leporini. Scaling between relaxation, transport, and caged dynamics in polymers: From cage restructuring to diffusion. J.Phys. Chem. B, 115:14046-14051, 2011.

[3] D. S. Simmons, M. T. Cicerone, Q. Zhong, M. Tyagi, and J. F. Douglas. Generalized localization model of relaxation in glass-forming liquids. Soft Matter, 8:11455-11461, 2012.

[4] F. Puosi, C. De Michele, and D. Leporini. Scaling between relaxation, transport and caged dynamics in a binary mixture on a per-component basis. J. Chem. Phys., 138:12A532, 2013.

[5] Emmanuel Guillaud, Laurent Joly, Dominique de Ligny, and Samy Merabia. Assessment of elastic models in supercooled water: A molecular dynamics study with the tip4p/2005f force field. The Journal of Chemical Physics, 147(1):014504, 2017.

[6] R. Horstmann and M. Vogel. Common behaviors associated with the glass transitions of water-like models. The Journal of Chemical Physics, 147(3):034505, 2017.

[7] Jack F. Douglas, Beatriz A. Pazmiño Betancourt, Xuhang Tong, and Hao Zhang. Localization model description of diffusion and structural relaxation in glass-forming cu-zr alloys. J. Stat. Mech.: Theory Exp., page 054048, 2016.

[8] F. Puosi, A. Pasturel, N. Jakse, and D. Leporini. Communication: Fast dynamics perspective on the breakdown of the stokes-einstein law in fragile glassformers. The Journal of Chemical Physics, 148(13):131102, 2018.

[9] A. Ottochian, C. De Michele, and D. Leporini. Universal divergenceless scaling between structural relaxation and caged dynamics in glass-forming systems. J. Chem. Phys., 131:224517, 2009.

[10] A. Ottochian and D. Leporini. Scaling between structural relaxation and caged dynamics in $\mathrm{ca}_{0.4} \mathrm{k}_{0.6}\left(\mathrm{no}_{3}\right)_{1.4}$ and glycerol: free volume, time scales and implications for the pressure-energy correlations. Phil. Mag., 91:1786-1795, 2011.

[11] F. Puosi and D. Leporini. Correlation of the instantaneous and the intermediate-time elasticity with the structural relaxation in glassforming systems. J. Chem. Phys., 136:041104, 2012.

[12] F. Puosi and D. Leporini. Communication: Fast and local predictors of the violation of the stokes- einstein law in polymers and supercooled liquids. J. Chem. Phys., 136:211101, 2012.

[13] Beatriz A. Pazmiño Betancourt, Paul Z. Hanakata, Francis W. Starr, and Jack F. Douglas. Quantitative relations between cooperative motion, emergent elasticity, and free volume in model glass-forming polymer materials. Proc. Natl. Acad. Sci. USA, 112:2966-2971, 2015.

[14] Matteo Becchi, Andrea Giuntoli, and Dino Leporini. Molecular layers in thin supported films exhibit the same scaling as the bulk between slow relaxation and vibrational dynamics. Soft Matter, 14:8814-8820, 2018.

[15] Karolina Adrjanowicz, Roksana Winkler, Andrzej Dzienia, Marian Paluch, and Simone Napolitano. Connecting $1 \mathrm{~d}$ and $2 \mathrm{~d}$ confined polymer dynamics to its bulk behavior via density scaling. ACS Macro Letters, 8(3):304-309, 2019.

[16] F Puosi, N Jakse, and A Pasturel. Dynamical, structural and chemical heterogeneities in a binary metallic glass-forming liquid. Journal of Physics: Condensed Matter, 30(14):145701, 2018.

[17] Elijah Flenner and Grzegorz Szamel. Fundamental differences between glassy dynamics in two and three dimensions. Nature Communications, 6(1):7392, dec 2015.

[18] Bernd Illing, Sebastian Fritschi, Herbert Kaiser, Christian L. Klix, Georg Maret, and Peter Keim. MerminWagner fluctuations in 2D amorphous solids. Proceedings of the National Academy of Sciences, 114(8):1856-1861, feb 2017.

[19] Steve Plimpton. Fast Parallel Algorithms for Short-Range Molecular Dynamics. Journal of Computational Physics, 117(1):1-19, mar 1995.

[20] M.I. Mendelev, M.J. Kramer, R.T. Ott, D.J. Sordelet, D. Yagodin, and P. Popel. Development of suitable interatomic potentials for simulation of liquid and amorphous $\mathrm{Cu} Z \mathrm{Zr}$ alloys. Philos. Mag., 89(11):967987, 2009. 
[21] Yuan-Chao Hu, Hajime Tanaka, and Wei-Hua Wang. Impact of spatial dimension on structural ordering in metallic glass. PHYSICAL REVIEW E, 96:22613, 2017.

[22] Hayato Shiba, Peter Keim, and Takeshi Kawasaki. Isolating long-wavelength fluctuation from structural relaxation in two-dimensional glass: cage-relative displacement. Journal of Physics: Condensed Matter, page aaa8b8, 2018.

[23] Skanda Vivek, Colm P Kelleher, Paul M Chaikin, and Eric R Weeks. Long-wavelength fluctuations and the glass transition in two dimensions and three dimensions. Proceedings of the National Academy of Sciences of the United States of America, 114(8):1850-1855, feb 2017.

[24] A. Rahman. Correlations in the motion of atoms in liquid argon. Phys. Rev., 136:A405-A411, Oct 1964.

[25] Hayato Shiba, Yasunori Yamada, Takeshi Kawasaki, and Kang Kim. Unveiling Dimensionality Dependence of Glassy Dynamics: 2D Infinite Fluctuation Eclipses Inherent Structural Relaxation. Physical Review Letters, 117(24):245701, dec 2016.

[26] Asaph Widmer-Cooper, Heidi Perry, Peter Harrowell, and David R. Reichman. Irreversible reorganization in a supercooled liquid originates from localized soft modes. Nature Physics, 4(9):711-715, sep 2008.

[27] Hao Zhang, Cheng Zhong, Jack F. Douglas, Xiaodong Wang, Qingping Cao, Dongxian Zhang, and JianZhong Jiang. Role of string-like collective atomic motion on diffusion and structural relaxation in glass forming Cu-Zr alloys. The Journal of Chemical Physics, 142(16):164506, apr 2015.

[28] F. Puosi, O. Chulkin, S. Bernini, S. Capaccioli, and D. Leporini. Thermodynamic scaling of vibrational dynamics and relaxation. The Journal of Chemical Physics, 145(23):234904, 2016.

[29] R. W. Hall and P. G. Wolynes. The aperiodic crystal picture and free energy barriers in glasses. J. Chem. Phys., 86:2943-2948, 1987.

[30] C. M. Roland, S. Hensel-Bielowka, M. Paluch, and R. Casalini. Supercooled dynamics of glass-forming liquids and polymers under hydrostatic pressure. Rep. Prog. Phys., 68:1405, 2005.

[31] M. C. C. Ribeiro, T. Scopigno, and G. Ruocco. Computer simulation study of thermodynamic scaling of dynamics of 2ca(no[sub 3])[sub 2] [center-dot] 3kno[sub 3]. J. Chem. Phys., 135:164510, 2011.

[32] S Bernini, F Puosi, and D Leporini. Thermodynamic scaling of relaxation: insights from anharmonic elasticity. Journal of Physics: Condensed Matter, 29(13):135101, feb 2017. 